\title{
Yanshu spraying agent, a traditional Chinese medicine, relieves chronic pharyngitis in animals by anti-inflammatory and antibacterial effects
}

\author{
CHENGWEN LU ${ }^{1}$, YANQIN SONG ${ }^{1}$, JIANQIAO ZHANG ${ }^{1}$, YUAN DU $^{1}$, TIAN WANG ${ }^{1}$, \\ YUNLI XUE $^{2}$, FENGHUA FU ${ }^{1}$ and LEIMING ZHANG ${ }^{1}$ \\ ${ }^{1}$ Department of Pharmacology, School of Pharmacy, Yantai University, Yantai, Shandong 264005; \\ ${ }^{2}$ Shandong Luye Pharmaceutical Company, Ltd., Yantai, Shandong 264003, P.R. China
}

Received September 2, 2013; Accepted January 21, 2014

DOI: $10.3892 / e t m .2014 .1524$

\begin{abstract}
Chronic pharyngitis is chronic inflammation that is often caused by repeated occurrences of acute pharyngitis or upper respiratory tract infections, including Streptococcus and Staphylococcus. The present study aimed to investigate the effects of Yanshu spraying agent (Yanshu) in relieving chronic pharyngitis, as well as the possible underlying mechanisms. The results revealed that Yanshu inhibited chronic inflammation in ammonia-induced chronic pharyngitis in rabbits and cotton pellet-induced granuloma tissue formation in rats. Yanshu also demonstrated antibacterial effects on Streptococcus and Staphylococcus in vitro. Yanshu did not exhibit any effects on the immune system, including the spleen and thymus indexes, immunocyte count and monocyte-macrophage function, when compared with the effects of dexamethasone. Therefore, the results of the present study indicate that Yanshu may relieve chronic pharyngitis via its anti-inflammatory and antibacterial activities.
\end{abstract}

\section{Introduction}

Chronic pharyngitis, a chronic inflammation of the pharyngeal mucous membrane and submucous lymphoid tissues, is often caused by unsatisfactory treatment of acute pharyngitis. The disease may also occur in response to chronic inflammation caused by alcohol abuse, overuse of the voice and cigarettes. Clinically, chronic pharyngitis manifests itself as itching, dryness, soreness of the throat, coughing and the feeling of a foreign body or obstruction in the throat (1). Chronic pharyngitis may have an infectious or non-infectious etiology. In bacterial pharyngitis, group A $\beta$-hemolytic

Correspondence to: $\mathrm{Dr}$ Leiming Zhang, Department of Pharmacology, School of Pharmacy, Yantai University, Qingquan Road 30, Yantai, Shandong 264005, P.R. China

E-mail: zhangleiming2009@126.com

Key words: chronic pharyngitis, Yanshu spraying agent, anti-inflammatory effects, antibacterial effects
Streptococcus and Staphylococcus aureus are most frequently $(5-36 \%)$ isolated $(2,3)$. Chronic pharyngitis may be caused by immune dysfunction of the body, as the immune system is insufficient in suppressing persistent microbial infection caused by bacteria, including $\beta$-hemolytic Streptococcus and Staphylococcus aureus (4).

Treatments for chronic pharyngitis vary depending on the underlying cause. Immediate treatment includes anti-inflammatory medications to reduce the swelling in the throat and improve the comfort of the patient, as well as antibiotic or antiviral medications that target infectious organisms in the throat. Yanshu spraying agent (Yanshu), a potential therapeutic agent for pharyngitis in clinical practice, consists of 11 herbs, including Radix Sophorae Tonkinensis, Calyx seu Fructus Physalis Francheti and Radix Scutellariae Baicalensis. A previous study demonstrated that Yanshu exhibits potent anti-inflammatory activity on the xylene-induced ear and carrageenan-induced paw edema models, which may be mediated by inhibiting the expression of cyclooxygenase-2 (5). However, the pharmacological effects of Yanshu on chronic pharyngitis have not yet been demonstrated. Thus, the aim of the present study was to investigate the effects of Yanshu on chronic pharyngitis in animals and the anti-inflammatory and antibacterial mechanisms.

\section{Materials and methods}

Drugs and chemicals. Yanshu was provided by Shandong Luye Pharmaceutical Co, Ltd. (20120525; Yantai, China). Yanshu is composed of 11 plant materials, including Radix Sophorae Tonkinensis, Calyx seu Fructus Physalis Francheti, Periostracum Cryptotympanae, Radix Rehmanniae, Fructus Arctii, Radix Scutellariae Baicalensis, Radix Paeoniae Rubra, Semen Qroxyli, Semen Sterculiae Lychnophorae, Cortex Moutan Radicis and Herba Menthae Haplocalycis, at a ratio of 1.7:1.7:1.7:2.4:1.7:1.7:2:1.7:1.7:1.7:1.7, respectively (dry weight). All plant materials were selected according to the pharmacopoeia of the People's Republic of China (2010) (6). Yanshu $(2 \mathrm{~kg})$ was prepared as a mixture of the aforementioned components and extracted with 24 and then 20 liters distilled water at $100^{\circ} \mathrm{C}$ for $1.5 \mathrm{~h}$, respectively. After filtering 
the liquid, the extract was concentrated with a rotary vacuum evaporator to reach a concentration of $2.0 \mathrm{~g} / \mathrm{ml}$ which was stored at $4^{\circ} \mathrm{C}$.

Diclofenac diethylamine emulsion (Votalin) was purchased from Beijing Novartis Pharma Co., Ltd (X0587; Beijing, China). Penicillin round paper (diameter, $6 \mathrm{~mm}$; content, $10 \mu \mathrm{g}$ ) was purchased from Hangzhou Tianhe Microorganism Reagent Co., Ltd (111209; Hangzhou, China) and dexamethasone was purchased from Shandong Lukang Cisen Pharmaceutical Co., Ltd (120229203; Jining, China).

Animals. Male Swiss mice (weight, 18-22 g) and male Wistar rats (weight, 160-200 g) were purchased from Beijing Vital River Laboratories Animals Technology Co., Ltd (Beijing, China). New Zealand white rabbits (weight, $2.5-3 \mathrm{~kg}$ ) were purchased from the Experimental Animal Center of Shandong Luye Pharmaceutical Co., Ltd. (Yantai, China). All experimental procedures in the study were performed in accordance with the guidelines for the Care and Use of Laboratory Animals from the National Institute of Health and were approved by the Ethics Committee of Yantai University (Yantai, China). All the animals were housed in diurnal lighting conditions (12:12 h) and provided with access to food and water ad libitum.

Chronic pharyngitis animal model. The chronic pharyngitis animal model was established as previously described (7), but with minor modifications. Briefly, rabbits were sprayed with $2.5 \%$ ammonia water into the pharynx mucosal twice per day (600 $\mu$ l total) for 15 consecutive days. On day $8,0.5 \mathrm{ml}$ oil of turpentine was injected into the pharynx mucosal of the rabbits. The rabbits were randomly divided into the control, model, watermelon frost (reference drug used in acute and chronic pharyngitis) and Yanshu low-, medium- and high-dose groups ( $n=5$ per group). Each treatment group received the respective treatment of vehicle, $200 \mathrm{mg}$ watermelon frost or $300 \mu \mathrm{l}$ Yanshu $(0.5,1$ or $2 \mathrm{~g} / \mathrm{ml})$ sprayed into the pharynx mucosal four times a day for 14 consecutive days. After $24 \mathrm{~h}$ following the last administration, animals were anesthetized and pharyngeal tissue was removed and fixed in $4 \%$ formalin. The rabbits were anesthetized with urethane $(1 \mathrm{~g} / \mathrm{kg})$. Tissues were sliced for hematoxylin and eosin staining to observe pathological differences between the groups under a light microscope (Olympus Co., Ltd., Beijing, China).

Cotton pellet-induced granuloma tissue formation test. The cotton pellet-induced granuloma tissue formation animal model was established as previously described (8), but with minor modifications. Briefly, rats were anesthetized with $350 \mathrm{mg} / \mathrm{kg}$ chloral hydrate and two sterilized cotton pellets, that weighed $20 \mathrm{mg}$ each, were implanted subcutaneously on each side of the nape through a small ventral incision in the nape of the rats. Following implantation of the cotton pellets, each rat treatment group ( $n=10$ per group) received topical treatment of vehicle, $100 \mathrm{mg}$ Votalin or $300 \mu \mathrm{l}$ Yanshu at $0.5,1$ or $2 \mathrm{~g} / \mathrm{ml}$, four times a day for seven consecutive days. Eight days following implantation of the cotton pellets, the animals were anesthetized with chloral hydrate. Each implanted cotton pellet was removed with the surrounding fibrovascular tissue and dried at $60^{\circ} \mathrm{C}$ for $12 \mathrm{~h}$. The dry weight was then measured and the net granuloma weight was calculated by minusing the original pellet weight from the dry pellet weight. The granuloma inhibition rate was calculated as follows: Granuloma inhibition rate $(\%)=1$ - (drug group average granuloma net weight)/(control group average granuloma net weight) x 100 .

Antibacterial test in vitro. Antibacterial tests were performed using the cylinder-plate method (9). A tested $200 \mu \mathrm{l}$ bacteria suspension ( $\beta$-hemolytic Streptococcus and Staphylococcus aureus; concentration, $1 \times 10^{7} / \mathrm{ml}$ ) was daubed by SS-Spreader (Egoan Technology Co., Ltd., Beijing, China) on culture medium (Staphylococcus was cultured using nutrient agar plate and $\beta$-hemolytic Streptococcus using blood plate) under aseptic conditions. The ready plates were dried at room temperature for $15 \mathrm{~min}$ and the cylinder-plates $(6 \times 8 \times 10 \mathrm{~mm})$ were placed on the medium. Normal saline $(200 \mu \mathrm{l})$ or varied concentrations of Yanshu $(2,1,0.5,0.25,0.125,0.0625$ and $0.03125 \mathrm{~g} / \mathrm{ml} ; 200 \mu \mathrm{l})$ were added to the cylinder, respectively. Plates were then stored at $4^{\circ} \mathrm{C}$ for $2 \mathrm{~h}$ and cultured at $37^{\circ} \mathrm{C}$ for $24 \mathrm{~h}$. The diameters of the effective inhibitory zone of Yanshu were measured.

Spleen index, thymus index and immunocyte count. In total, 50 mice were randomly divided into vehicle, dexamethasone ( $5 \mathrm{mg} / \mathrm{kg}$ body weight per day) and three Yanshu groups (10, 20 and $40 \mathrm{~g} / \mathrm{kg}$ body weight per day; $\mathrm{n}=10$ per group). Mice received oral administration of the corresponding drugs once per day for seven consecutive days. After $24 \mathrm{~h}$ following the last administration, blood samples were collected for leukocyte and lymphocyte counts. Mice were then sacrificed and the weight of the spleen and thymus were measured. The index was calculated as follows: Organ index = organ weight (mg)/body weight $(\mathrm{g})$.

Carbon particle clearance test. Phagocytosis rates of monocytes and macrophages were estimated by calculating the speed of carbon particle clearance. The carbon particle clearance test was performed as previously described (1), but with minor modifications. Briefly, 50 mice were randomly divided into a vehicle, dexamethasone $(5 \mathrm{mg} / \mathrm{kg}$ body weight per day) and three Yanshu groups $(10,20$ and $40 \mathrm{~g} / \mathrm{kg}$ body weight per day; $n=10$ per group). Mice received oral administration of the corresponding drugs once a day for seven consecutive days. After $2 \mathrm{~h}$ following the last administration, Indian ink (0.1 ml/10 g; Beijing Xizhong Chemical Co., Ltd., Beijing, China) was injected intravenously and $20 \mu$ l blood samples were collected following $3 \mathrm{~min}$ and $13 \mathrm{~min}$. The blood samples were hemolyzed in $1 \mathrm{ml} \mathrm{Na} \mathrm{CO}_{3}(0.1 \%)$ and the concentration of carbon particles was determined by measuring the optical density (OD) at $600 \mathrm{~nm}$. The clearance index (CI) and phagocytic index $(\mathrm{PI})$ were calculated as follows: $\mathrm{CI}(\mathrm{K})=\left[\log \mathrm{OD}_{1}\right.$ (control) $-\log \mathrm{OD}_{2}$ (treated) $] / \mathrm{t}_{1}-\mathrm{t}_{2}$; and $\mathrm{PI}=$ body weight $/($ liver weight + spleen weight) $\mathrm{x}^{3} \sqrt{\mathrm{k}}$.

Statistical analysis. Data are expressed as the mean \pm SD. Data were analyzed using one-way analysis of variance with the Bonferroni post hoc test used for multiple t-tests. $\mathrm{P}<0.05$ was considered to indicate a statistically significant difference. 
A

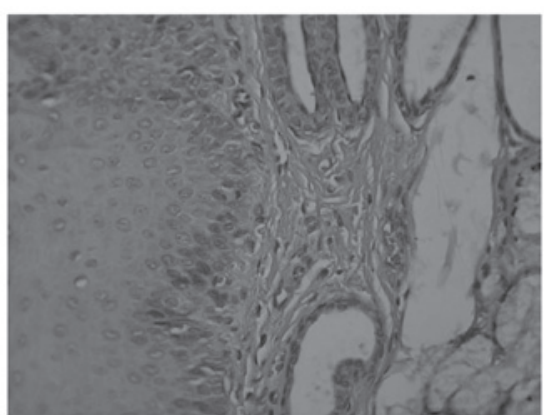

C

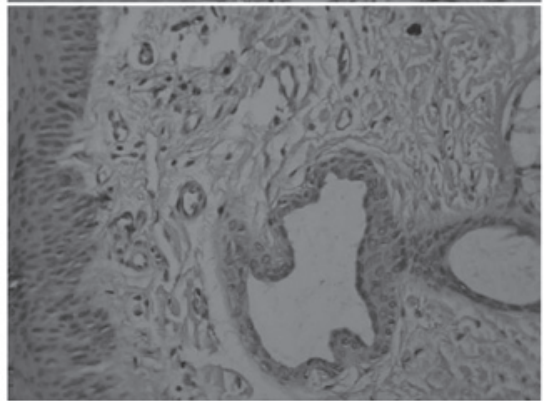

$\mathbf{E}$

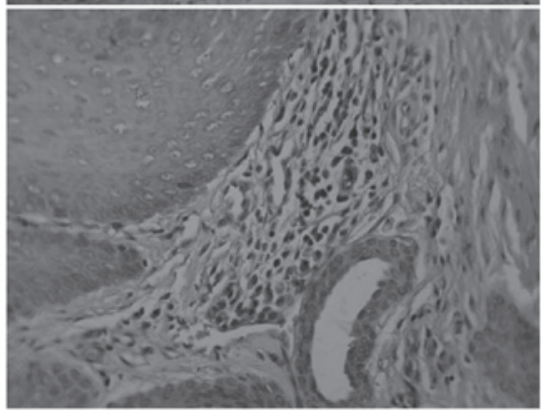

B

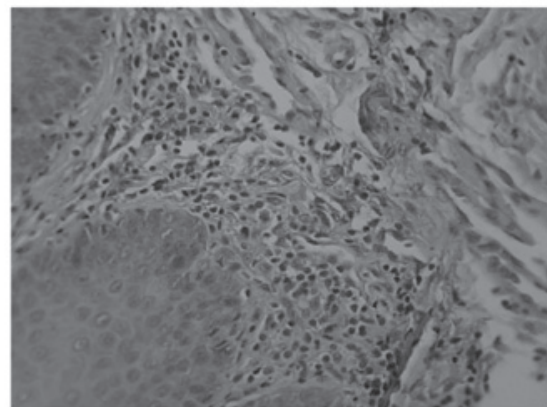

D

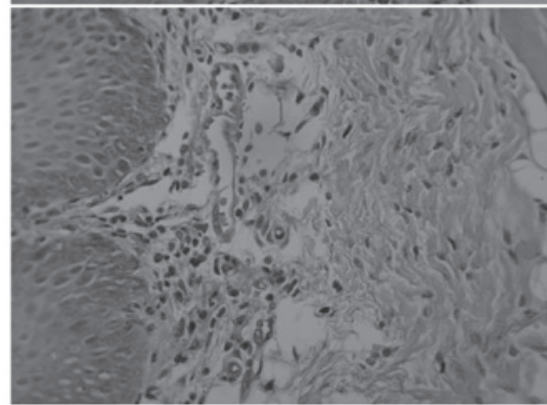

$\mathbf{F}$

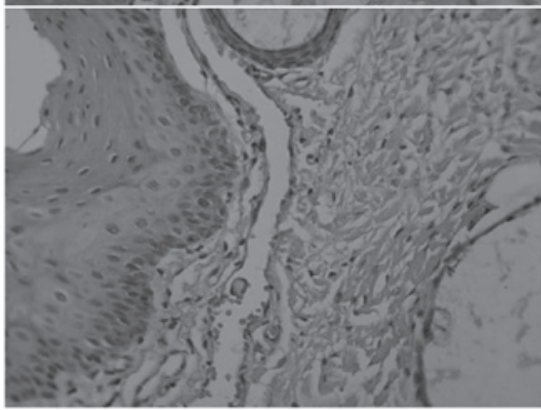

Figure 1. Histopathology of the pharyngeal tissue induced by 2.5\% ammonia in rabbits in (A) control, (B) model, (C) watermelon frost, (D) Yanshu low-dose, (E) Yanshu medium-dose and (F) Yanshu high-dose groups.

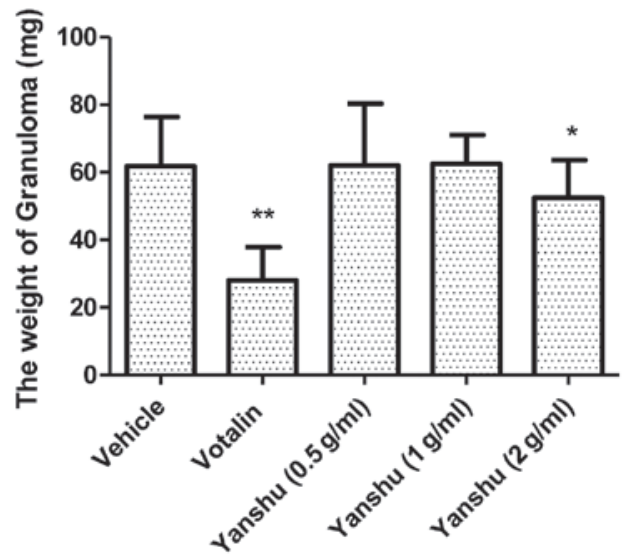

Figure 2. Effects of Yanshu on chronic granuloma inflammation in rats, as measured by granuloma weight. Results are expressed as the mean \pm SD $(\mathrm{n}=10) .{ }^{*} \mathrm{P}<0.05$ and $^{* * *} \mathrm{P}<0.01$, vs. vehicle group.

\section{Results}

Effect of Yanshu on chronic pharyngitis in rabbits. Under a light microscope, a large number of inflammatory cells were observed in the pharyngeal tissue following administration of $2.5 \%$ ammonia and the number of lymphocytes increased significantly. Compared with the model group, the watermelon frost (reference drug) and Yanshu high-dose treatment groups showed a significantly reduced number of inflammatory cells (Fig. 1).

Effect of Yanshu on chronic granulomatous inflammation in rats. When treated with Votalin or high-dose Yanshu, the granuloma weight decreased significantly $(\mathrm{P}<0.01$ and $\mathrm{P}<0.05$, respectively) as compared with the model group (Fig. 2).

Antibacterial activity of Yanshu in vitro. Yanshu demonstrated antibacterial activity against Staphylococcus aureus $(\geq 0.125 \mathrm{~g} / \mathrm{ml})$ and $\beta$-hemolytic Streptococci $(\geq 1 \mathrm{~g} / \mathrm{ml})$. The results are shown in Table I.

Effect of Yanshu on immune organsandimmune cells. Compared with the vehicle group, the thymus and spleen indexes in the Yanshu-treated mice did not change significantly. However, the dexamethasone-treated mice exhibited a significant decrease in thymus and spleen indexes $(\mathrm{P}<0.01$; Fig. $3 \mathrm{~A})$. There were no marked changes in the leukocyte and lymphocyte concentrations of mice treated with 10,20 and $40 \mathrm{~g} / \mathrm{kg}$ Yanshu, but treatment with dexamethasone resulted in a significant decrease in the number of lymphocytes ( $\mathrm{P}<0.01 ;$ Fig. 3B).

Effect of Yanshu on the phagocytic index of monocyte and macrophages in mice. The phagocytic index in 
Table I. Antibacterial activity of Yanshu in vitro (cylinder-plate method).

Diameter of inhabiting circles $(\mathrm{mm})$

Drug

Staphylococcus aureus

$\beta$-hemolytic Streptococcus

Saline

Penicillin $10 \mu \mathrm{g}$

$44.3 \pm 1.6$

$19.3 \pm 0.6$

Yanshu $2 \mathrm{~g} / \mathrm{ml}$

$10.7 \pm 0.6$

$7.5 \pm 0.5$

Yanshu $1 \mathrm{~g} / \mathrm{ml}$

$8.7 \pm 0.6$

$6.7 \pm 0.6$

Yanshu $0.5 \mathrm{~g} / \mathrm{ml}$

$7.7 \pm 1.2$

Yanshu $0.25 \mathrm{~g} / \mathrm{ml}$

$8.7 \pm 0.6$

Yanshu $0.125 \mathrm{~g} / \mathrm{ml}$

$8.3 \pm 0.6$

Yanshu $0.0625 \mathrm{~g} / \mathrm{ml}$

Yanshu $0.03125 \mathrm{~g} / \mathrm{ml}$

Values are the mean of triplicate experiments and '-' indicates no activity.

A

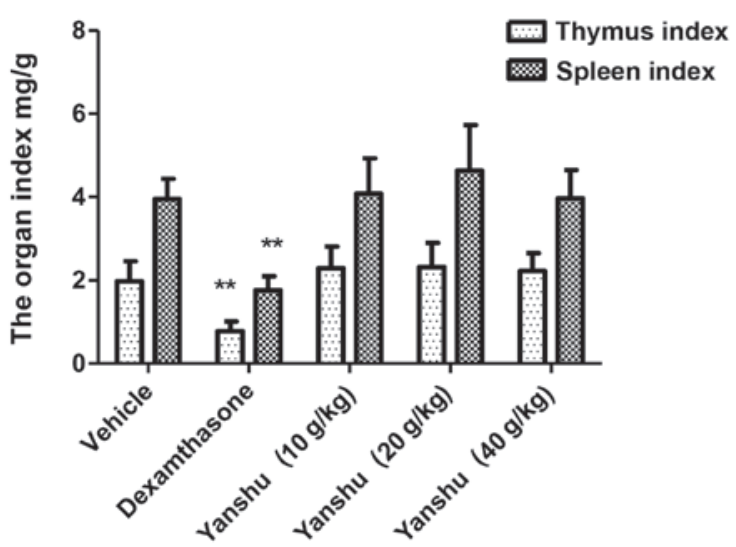

B

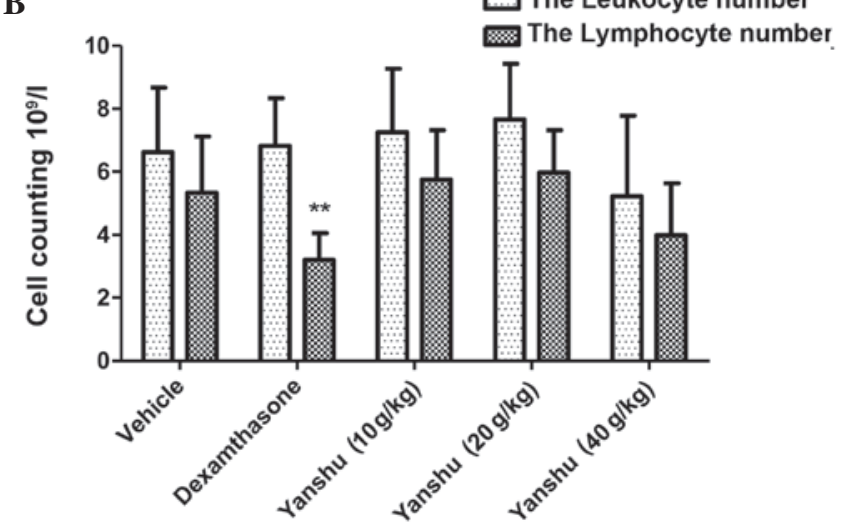

Figure 3. Effects of Yanshu on the (A) thymus and spleen indexes and (B) number of leukocytes and lymphocytes in mice. Results are expressed as the mean $\pm \mathrm{SD}(\mathrm{n}=10){ }^{* * *} \mathrm{P}<0.01$, vs. vehicle group.

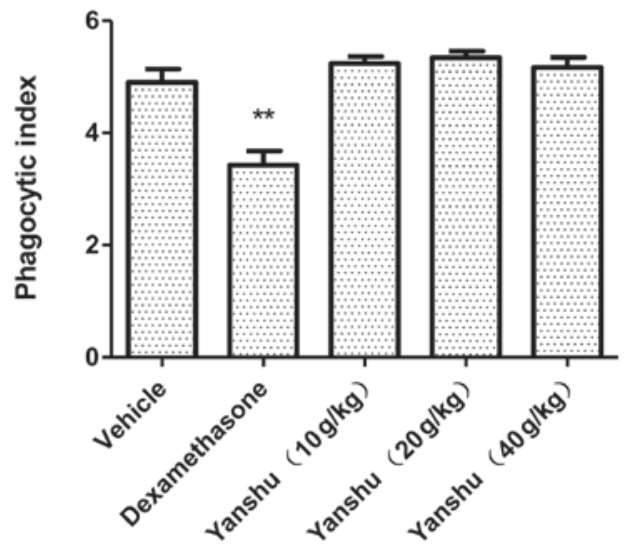

Figure 4. Effect of Yanshu on carbon particle clearance in mice. Results are expressed as the mean $\pm \mathrm{SD}(\mathrm{n}=10)$. ${ }^{* *} \mathrm{P}<0.01$, vs. vehicle group.

Yanshu-treated mice did not change significantly compared with the vehicle group. However, dexamethasone-treated mice exhibited a significant decrease in the phagocytic index ( $\mathrm{P}<0.01$; Fig. 4).

\section{Discussion}

The present study investigated the anti-inflammatory and antibacterial effects of Yanshu in relieving chronic pharyngitis, as well as the effect Yanshu has on the immune system. The results demonstrated that Yanshu inhibits chronic inflammation in animals and demonstrates antibacterial effects in vitro. As an anti-inflammatory drug, Yanshu does not affect immune function as compared with dexamethasone.

Chronic pharyngitis is chronic inflammation of the pharyngeal mucous membrane. In the present study, an ammonia-induced rabbit chronic pharyngitis model was established $(10,11)$ to investigate the anti-inflammatory effects of Yanshu. The results revealed that Yanshu suppresses ammonia-induced chronic inflammation in pharyngeal tissue. Similar results were also observed in the cotton pellet-induced granulomatous inflammation model (12). The results indicate that Yanshu exhibits inhibitory effects against chronic inflammation.

Bacterial infection, particularly $\beta$-hemolytic Streptococcus infection, is one of the etiologies of pharyngitis $(13,14)$. To investigate the antibacterial effects of Yanshu, the 
cylinder-plate method was used. This is a sensitive and popular method for antibacterial experiments and by measuring the diameter of the inhibition zones, the antibacterial effects of Yanshu were analyzed. Treatment with Yanshu significantly inhibited the growth of $\beta$-hemolytic Streptococcus and Staphylococcus aureus. Therefore, the results indicate that Yanshu exerts good antibacterial effects.

Pharyngitis is often a sign of immune dysfunction, as the immune system is usually robust enough to prevent throat infections. Chronic pharyngitis may be caused by immune dysfunction, as the immune system is insufficient in suppressing persistent infections, including Staphylococcal and Streptococcal infections. Therefore, treatment for pharyngitis needs to enhance immune function or at least not suppress function, unlike glucocorticoids that are commonly administered for pharyngitis (15-17). In the present study, Yanshu was found to have no inhibitory effects on immune function when compared with dexamethasone.

In conclusion, the present study demonstrated that Yanshu exhibits anti-inflammatory and antibacterial effects in vivo. In addition, Yanshu did not exhibit significant effects on the immune system in vitro. These observations support the use of Yanshu as a safe therapeutic agent for the treatment of chronic pharyngitis.

\section{Acknowledgements}

The study was supported by the 11th Five Years Key Programs for Science and Technology Development of China (grant no. 2010ZX09102-207) and the Taishan Scholar Project. The authors thank Professor Tongshen Liu for providing technical assistance in the pathological observations.

\section{References}

1. Sun Y, Zang Z, Xu X, Zhang Z, Zhong L, Zan W, Zhao Y and Sun L: Experimental investigation of the immunoregulatory and anti-inflammatory effects of the traditional Chinese medicine 'Li-Yan Zhi-Ke Granule' for relieving chronic pharyngitis in rats. Mol Biol Rep 38: 199-203, 2011.

2. Whiley RA and Beighton D: Emended description and recognition of Streptococcus constellatus, Streptococcus intermedius, and Streptococcus anginosus as distinct species. Int J Syst Bacteriol 41: 1-5, 1991.
3. Belko J, Goldmann DA, Marcone A and Zaidi AK: Clinically significant infections with organisms of the Streptococcus milleri group. Pediatr Infect Dis J 21: 715-723, 2002.

4. Margarino G, Scala M, Rossi E, Mereu P, Dessy G, Sogno G, Bonelli $L$ and Badellino F: Immunologic study of patients with chronic pharyngitis. Acta Otorhinolaryngol Ital 9: 399-402, 1989 (In Italian).

5. Zhang J, Wang H, Wang T, Chong Y, Yu P, Lu C, Xue Y, Fu F and Zhang L: Anti-inflammatory activity of Yanshu spraying agent in animal models. Exp Ther Med 5: 73-76, 2013.

6. Chinese Pharmacopoeia Commission (eds): Pharmacopoeia of the People's Republic of China. Beijing: Chinese Medical Science and Technology Press, 2010.

7. Fan KH: Influence of Yanyanling granules on the morphopathology of chronic pharyngitis in rabbits. Hua Xi Yao Xue Za Zhi 28: 60-61, 2013 (In Chinese).

8. Moreira LQ, Vilela FC, Orlandi L, Dias DF, Santos AL, da Silva MA, Paiva R, Alves-da-Silva G and Giusti-Paiva A: Anti-inflammatory effect of extract and fractions from the leaves of Byrsonima intermedia A. Juss. in rats. J Ethnopharmacol 138: 610-615, 2011.

9. Breier AR, Garcia CV, Oppe TP, Steppe M and Schapoval EE: Microbiological assay for azithromycin in pharmaceutical formulations. J Pharm Biomed Anal 29: 957-961, 2002.

10. Jia WX, Xu QR, Ren XH, He N, Zhao Y, Wang LJ, Yang LH and Yu ZY: Effects of Jinhoujian spraying agent for the expression of interleukin-1 and 125I-EGF of chronic pharyngitis. Hebei Yi Yao 32: 2697-2698, 2010 (In Chinese).

11. Zhong Q, Peng SL and Li X: The research of Yinxuanye for chronic pharyngitis. Shandong Zhong Yi Za Zhi 19: 99-100, 2000 (In Chinese).

12. Barreto RR, Franco Ede S, Brasileiro CF, de Oliveira AP, Dimech GS, Malta DJ, Cazuzu JS, Leite AC, da Silva TG and Maia MB: Early undernutrition is associated with attenuated inflammatory response and alteration in pharmacological efficacy of indomethacin in rats. Eur J Pharm Sci 46: 56-63, 2012.

13. Murray RC and Chennupati SK: Chronic streptococcal and non-streptococcal pharyngitis. Infect Disord Drug Targets 12: 281-285, 2012.

14. Choby BA: Diagnosis and treatment of streptococcal pharyngitis. Am Fam Physician 79: 383-390, 2009.

15. Kumari JO and Rajendran R: Effect of topical nasal steroid spray in the treatment of non-specific recurrent/chronic pharyngitis - a trial study. Indian J Otolaryngol Head Neck Surg 60: 199-201, 2008.

16. Sadowitz PD, Page NE and Crowley K: Adverse effects of steroid therapy in children with pharyngitis with unsuspected malignancy. Pediatr Emerg Care 28: 807-809, 2012.

17. Wing A, Villa-Roel C, Yeh B, Eskin B, Buckingham J and Rowe BH: Effectiveness of corticosteroid treatment in acute pharyngitis: a systematic review of the literature. Acad Emerg Med 17: 476-483, 2010. 